\title{
Sex-based differences in the morphometric parameters of distal of the femur: A magnetic resonance imaging study
}

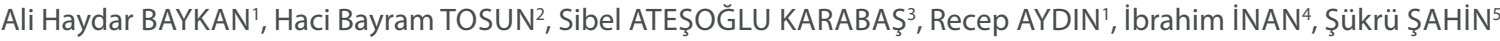 \\ ${ }^{1}$ Department of Radiology, Faculty of Medicine, Adiyaman University, Adiyaman, Turkey \\ 2 Department of Orthopaedics and Traumatology, Faculty of Medicine, Adiyaman University, Adiyaman, Turkey \\ ${ }^{3}$ Department of Anatomy, Faculty of Medicine, Adiyaman University, Adiyaman, Turkey \\ ${ }^{4}$ Department of Radiology, Biruni University Hospital, Istanbul, Turkey \\ ${ }^{5}$ Departmenti of Radiology, Adiyaman Research and Educational Hospital, Adiyaman, Turkey \\ Correspondence \\ Ali Haydar BAYKAN \\ Adıyaman Üniversitesi Tıp Fakültesi Radyoloji Anabilim Dalı, 02100 Adıyaman, Türkiye \\ e-mail:drbaykan@yahoo.com
}

\section{ABSTRACT}

The morphology of the knee joint varies according to sex and ethnic groups. Full knowledge of these variable morphological data is important for achieving formal compatibility between the knee surface to be resected and the prosthesis when designing knee arthroplasty. This study aimed to perform morphometric measurements of distal femoral anatomy in healthy adults in terms of sex and age.

The magnetic resonance imaging (MRI) images of 180 patients aged 22 years or older were retrospectively examined in 2018. The anthropometric measurements of the distal femoral anatomy were performed using proton density spectral attenuated inversion recovery MRI sequences. The anatomic and surgical transepicondylar axis length, anterolateral and anteromedial condylar depth, posterior condylar length, epicondylar axis angle, intercondylar notch width and length, and medial and lateral condylar height were measured in all cases. The values obtained were recorded in millimeters and degrees and evaluated through comparisons by sex and age.

The distal femoral dimensions were significantly higher in males than in females in terms of all parameters measured independently of age $(P<0.05)$.

In this study, the normal reference values of distal femur measurements were determined using MRI in adult patients. These reference values can guide the knee surgery.

Key words: Knee, magnetic resonance imaging, morphometry

\section{INTRODUCTION}

Knee osteoarthritis is an arthropathy with a chronic and progressive course that negatively affects the daily activities of individuals, decreases their quality of life, and may result in the loss of articular cartilage at older ages (1-3). Total knee arthroplasty (TKA) is a good treatment option for patients with osteoarthritis suffering from secondary knee pain, who do not respond to conservative treatment. TKA is a reliable procedure that improves patients' functional status and relieves pain (3). Accurate bone-cutting, soft tissue balance, and adequate covering of the resected surface with the implant are important factors affecting successful outcomes in THA $(4,5)$. Anatomical dif- ferences between sexes have been discussed in recent studies on TKA surgery (6). It is recommended that new prosthetic designs should be developed to ensure compliance with these anatomical differences (7). The anthropometric data are very important for the stability and long life of the implants to be used in TKA. TKA is expected to yield better results based on fully measured morphological data of the knee, morphological differences between sexes, and morphological compatibility between the tibia and the femur (8).

This study aimed to perform morphometric measurements of distal femoral anatomy in healthy adults. 


\section{MATERIALS AND METHODS}

The study was carried out with the approval of the Clinical Ethics Committee of Adiyaman University and the working principles of the Helsinki Declaration. The magnetic resonance imaging (MRI) images of patients aged 22 years and older, who underwent an MRI examination between June and December 2018, were obtained from the electronic patient registry system of the hospital and retrospectively analyzed.

A total of 180 patients with normal healthy knees exhibiting no pathology on knee MRI were included in the study. The patients were divided into the following three age groups, each containing 30 men and 30 women: $22-40$ years (Group 1), 41-50 years (Group 2), and $51-60$ years (Group 3). The sex and anthropometric measurements were comparatively evaluated between the three groups.

\section{MRI protocol}

All MRI procedures were performed using a 1.5T system (Achieva; Philips Medical Systems, Best, the Netherlands) using a knee coil. Contrast material was not used. Sequences used for knee MRI examination were proton density spectral attenuated inversion recovery (PD-SPAIR) coronal and axial MRI sequences.

Coronal plane parameters: Repetition time (TR), $3000 \mathrm{~ms}$; echo time (TE), $30 \mathrm{~ms}$; field of view (FOV), $160 \times 180 \mathrm{~mm}^{2}$; matrix, $244 \times 192 \mathrm{~mm}^{2}$; number of signal averages (NSA), 2; slice thickness, $3 \mathrm{~mm}$; and gap, $0.5 \mathrm{~mm}$.

Axial plane parameters: TR, $3000 \mathrm{~ms} ; \mathrm{TE}, 30 \mathrm{~ms}$; FOV, $160 \times 170 \mathrm{~mm}^{2}$; matrix, $244 \times 192 \mathrm{~mm}^{2}$; NSA, 2; slice thickness, $3 \mathrm{~mm}$; and gap, $0.5 \mathrm{~mm}$.

\section{Image analysis and measurements}

The measurements were performed by a single, experienced radiologist using the same device to increase reliability. The values obtained were recorded in millimeters and degrees.

Anatomical transepicondylar axis (aTEA) length: Measured from the mediolateral line connecting the outermost edge of the epicondyle in the axial plane (Fig. 1).

Surgical epicondylar axis (sTEA) length: Measured from the mediolateral line connecting the most protruding point of the epicondyle and the medial sulcus in the axial plane (Fig. 1).
Anterolateral condylar surface depth (ALCSD) and anteromedial condylar surface depth (AMCSD): The lines perpendicular to the surgical epicondylar axis and passing between two condyles measured from the mediolateral line extending parallel to the surgical epicondylar axis at the deepest portion of the trochlear groove in the axial plane (Fig. 1).

Posterior condylar line length (PCL): The mediolateral length passing between the two posterior condyles in the axial plane (Fig. 1).

Epicondylar axis angle (EPAA): The angle between the anatomical epicondylar axis and the surgical epicondylar axis in the axial plane (Fig. 2).

Intercondylar notch width (NW): Mediolateral length at the widest part of the section where the entire intercondylar fossa was visualized in the axial plane (Fig. 3).

Intercondylar notch length (NL): Craniocaudal length at the deepest part of the section where the entire intercondylar fossa was visualized in the coronal plane (Fig. 4).

Medial and lateral condylar height (MCH and LCH): Craniocaudal length at the highest section where the entire intercondylar fossa was visualized in the coronal plane (Fig. 5).

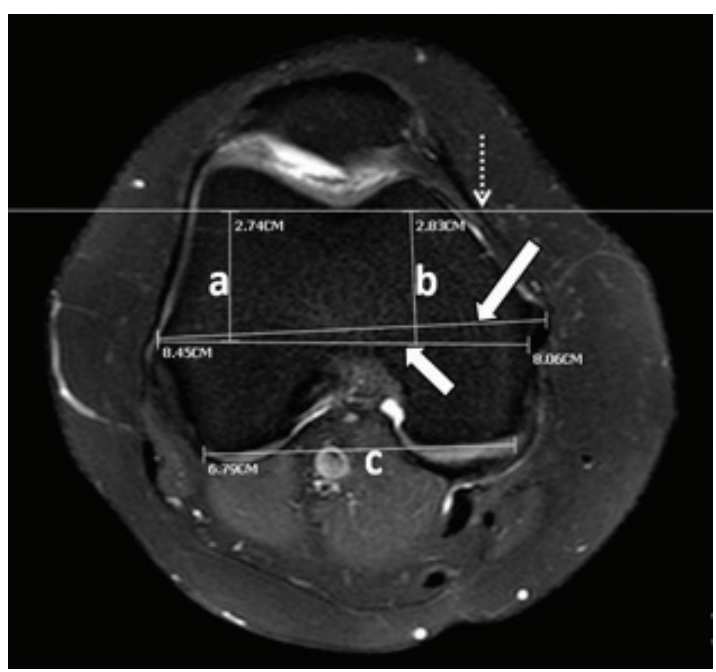

Figure 1: Axial PD-SPAIR image: Surgical transepicondylar axis length (short arrow) and anatomical transepicondylar axis length (long arrow). The anterolateral (a) and (b) anteromedial condylar surface depth and posterior condylar length (c) were measured from the deepest portion of the trochlear groove on the reference line (dashed arrow) running parallel to the surgical transepicondylar line. 


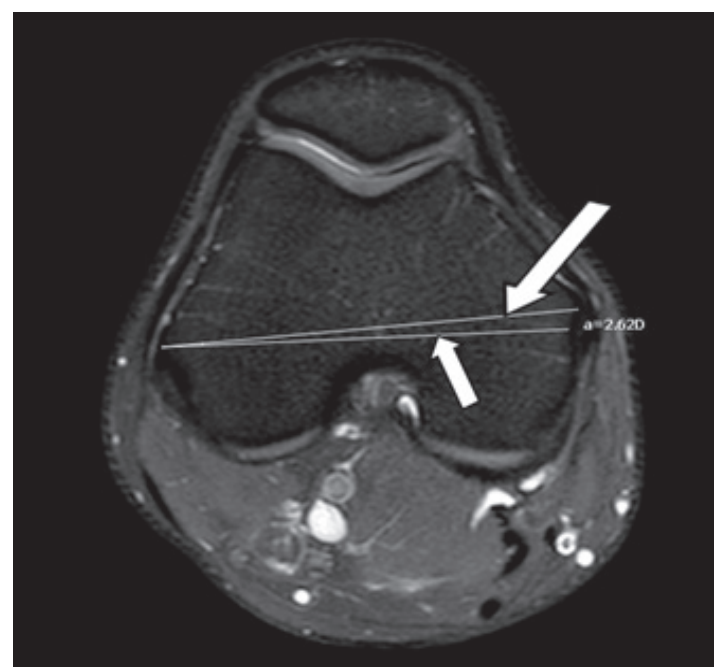

Figure 2: Axial PD-SPAIR image: Epicondylar angle a) between the surgical transepicondylar line (short arrow) and b) anatomical transepicondylar line (long arrow).

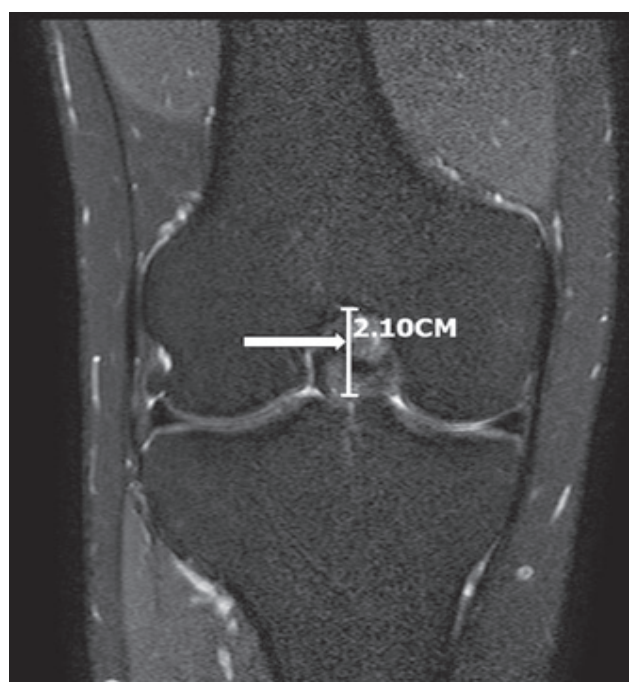

Figure 4: Coronal PD-SPAIR image: Intercondylar notch length (arrow).

\section{Statistical analysis}

Statistical analysis was performed using SPSS v. 15.0 (IL, USA). The normal distribution of variables and groups was evaluated using the Kolmogorov-Smirnov test. The independent two-sample t test was used to determine the differences between variables and sexes. One-way analysis of variance was used for the comparison between the variables and age groups. The Levene test was performed to check the homogene-

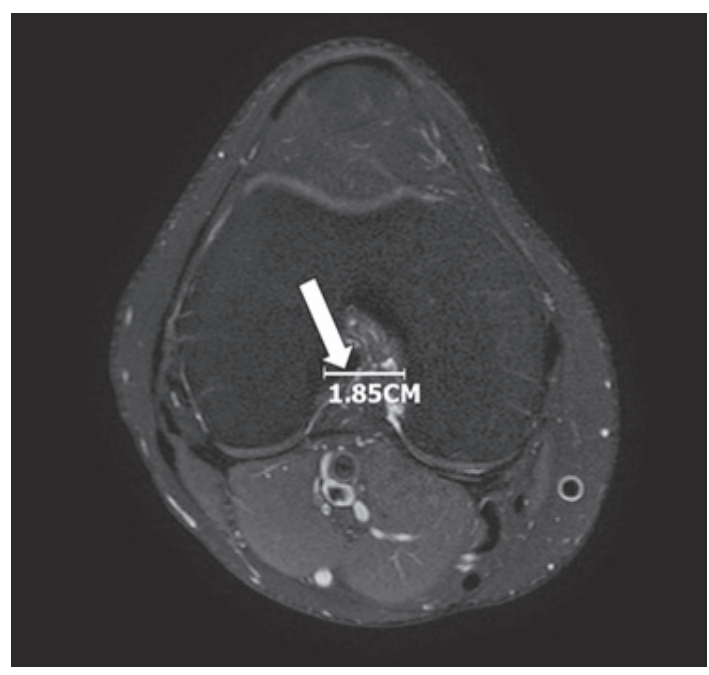

Figure 3: Axial PD-SPAIR image: Intercondylar notch width (arrow).

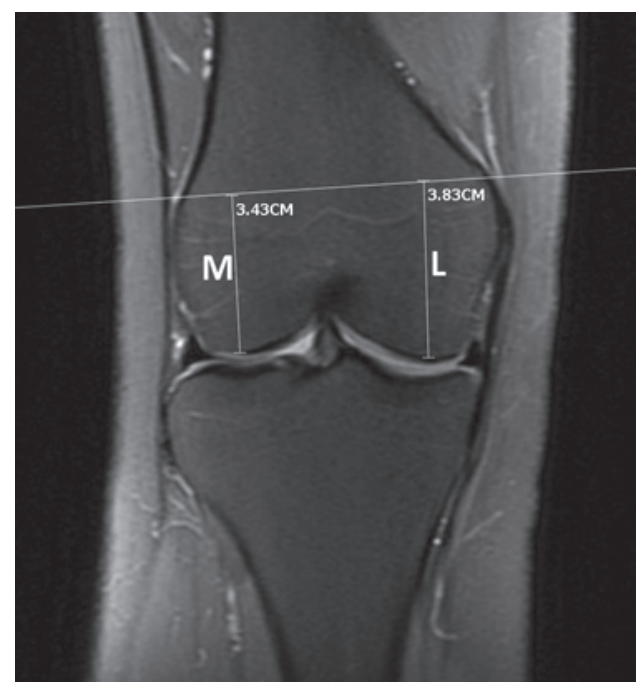

Figure 5: Coronal PD-SPAIR image. Medial (M) and lateral (L) condylar lengths.

ity of variances. To determine the differences between the significant variables and groups, Tukey's pairedcomparison analysis was performed. The results were given as mean \pm standard deviation. The minimum significance level was considered as $P<0.05$.

\section{RESULTS}

The median age was calculated as $28.5 \pm 4.95$ years for Group 1, $44 \pm 2.93$ years for Group 2, and 55 \pm 2.36 years for Group 3 . 
In groups formed according to age distributions, the ALCSD, AMCSD, NW, MCH, EPAA, and LCH values were significantly higher in Group $1(P<0.05)$ compared with Groups 2 and 3, whereas no significant difference was found between Group 2 and Group 3 in terms of these parameters $(P>0.05)$. No statistically significant difference was found between the three groups in terms of aTEA and sTEA lengths, $P C L$, and NL $(P>0.05)$ (Table 1).

When the morphometric measurements were evaluated within the groups according to sex, ALCSD, AMCSD, PCL, and aTEA and STEA lengths were found to be statistically significantly higher in men than in women in all three groups $(P<0.05)$. In Group 1, NW was significantly higher in men than in women $(P<0.05)$ while in Groups 2 and 3, no significant difference was observed between sexes $(P>0.05)$. In Group 2, NL was significantly higher in men than in women $(P<0.05)$, but no significant difference was noted between Group 1 and Group $3(P>0.05)$. In Groups 1 and 2, MCH and LCH were significantly greater in men than in women $(P<0.05)$; however, no significant sex-related difference was observed in Group $3(P>0.05)$. In Group 3, EPAA was significantly greater in men than in women $(P<0.05)$ whereas no significant difference was detected between sexes in the remaining groups $(P>0.05)$ (Table 2$)$.

While evaluating the parameters in all cases according to sex independently of age, the distal femoral dimensions were found to be significantly larger in men than in women. Statistically significant differences were noted between men and women in terms of aTEA and sTEA lengths, ALCSD, AMCSD, PCL, NW, $\mathrm{NL}, \mathrm{EPAA}, \mathrm{MCH}$, and $\mathrm{LCH}$ in all cases regardless of age $(P<0.05)$ (Table 3).

\section{DISCUSSION}

Morphology of the knee joint is an entity that varies between ethnic groups. Morphotype and sex of patients are among the factors contributing to these variations (9). The most important factor for the long-term success in TKA is achieving a complete formal compatibility between the resected knee surface and the prosthesis (5). Proper placement of appropriately sized prosthetic components in the correct position is very important for the long-term survival of TKA (10). Anthropometric characteristics are closely related to genetic characteristics, environmental factors, sociocultural status, lifestyle, health, and functional status (10).

Merchant et al. reported similar or better postTKA outcomes for women compared with men, noting that morphological differences based on sex were not reflected in clinical outcomes (7). However, Cheng et al. suggested that morphological data could serve as a guide in the design of prostheses, and sex-based designs would be the most appropriate (8). Guy et al. determined a significant difference in the distal femoral morphology of men and women, with a considerable number of female cases exhibiting a significant overhang of the femoral component of a standard prosthesis. It was necessary to conduct long-term studies to measure the clinical effect of this situation on patients, indicating that a sex-specific implant might reduce potential medial-lateral (ML) overhang (11). The anthropometric measurements showed that the distal femoral dimensions were significantly smaller in women than in men.

In their CT study evaluating knees with osteoarthritis in an Arab population, Hafez et al. found that the distal femoral ML width was $72.04 \pm 6.6 \mathrm{~mm}$ and

Table 1: Measurement values of the distal femur according to the age groups.

\begin{tabular}{lllllllllll}
\hline & $\mathrm{NW}$ & $\mathrm{NL}$ & $\mathrm{MCH}$ & $\mathrm{LCH}$ & $\mathrm{PCL}$ & $\mathrm{aTEA}$ & $\mathrm{sTEA}$ & EPAA & ALCSD & AMCSD \\
\hline Groups 1 & $22.6 \pm 2.4$ & $22.8 \pm 2.2$ & $49.1 \pm 4.8$ & $50.9 \pm 4.2$ & $64.1 \pm 6.0$ & $80.7 \pm 7.4$ & $78.7 \pm 7.0$ & $3.65 \pm 0.81$ & $27.0 \pm 3.7$ & $27.6 \pm 3.8$ \\
Groups 2 & $21.1 \pm 2.9$ & $22.0 \pm 2.3$ & $45.9 \pm 5.5$ & $48.2 \pm 5.8$ & $64.9 \pm 6.4$ & $81.9 \pm 7.7$ & $80.0 \pm 7.3$ & $3.37 \pm 0.45$ & $25.8 \pm 3.4$ & $25.9 \pm 3.0$ \\
Groups 3 & $21.2 \pm 2.4$ & $22.2 \pm 2.2$ & $45.3 \pm 5.9$ & $47.6 \pm 5.9$ & $64.0 \pm 5.1$ & $81.1 \pm 6.7$ & $78.8 \pm 6.4$ & $3.45 \pm 0.58$ & $24.9 \pm 2.3$ & $25.1 \pm 2.3$ \\
$\mathrm{P}^{*}$ & $0.002^{*}$ & 0.139 & $<0.001^{*}$ & $0.002^{*}$ & 0.624 & 0.684 & 0.506 & $0.05^{*}$ & $0.001^{*}$ & $<0.001^{*}$ \\
\hline
\end{tabular}

${ }^{*} P<0.05$, Statistically significant. 
Table 2: Morphometric measurements of the groups according to sex.

\begin{tabular}{|c|c|c|c|c|c|c|c|c|c|c|c|}
\hline Groups & $\begin{array}{l}\text { Sex and } \\
\text { P value }\end{array}$ & NW & $\mathrm{NL}$ & $\mathrm{MCH}$ & $\mathrm{LCH}$ & $\mathrm{PCL}$ & aTEA & sTEA & EPAA & ALCSD & AMCSD \\
\hline \multirow{3}{*}{$\begin{array}{l}\text { Group } 1 \\
n=60\end{array}$} & Women & $21.9 \pm 2.1$ & $22.5 \pm 2.1$ & $46.2 \pm 2.7$ & $48.7 \pm 2.9$ & $60.6 \pm 5.6$ & $75.9 \pm 6.8$ & $74.3 \pm 6.6$ & $3.70 \pm 0.87$ & $24.7 \pm 3.2$ & $25.2 \pm 3.2$ \\
\hline & Men & $23.3 \pm 2.4$ & $23.1 \pm 2.2$ & $51.9 \pm 4.8$ & $53.1 \pm 4.2$ & $67.6 \pm 4.0$ & $85.5 \pm 4.1$ & $83.1 \pm 3.9$ & $3.59 \pm 0.73$ & $29.3 \pm 2.7$ & $29.9 \pm 2.8$ \\
\hline & $\mathrm{P}^{*}$ & $0.021^{*}$ & $0.340^{*}$ & $<0.001^{*}$ & $<0.001^{*}$ & $<0.001^{*}$ & $<0.001^{*}$ & $<0.001^{*}$ & 0.602 & $<0.001^{*}$ & $<0.001^{*}$ \\
\hline \multirow{3}{*}{$\begin{array}{c}\text { Group } 2 \\
n=60\end{array}$} & Women & $20.4 \pm 2.6$ & $21.4 \pm 2.3$ & $43.7 \pm 5.2$ & $45.7 \pm 5.2$ & $61.1 \pm 5.0$ & $76.7 \pm 6.1$ & $75.0 \pm 5.7$ & $3.43 \pm 0.50$ & $24.1 \pm 3.1$ & $24.2 \pm 2.6$ \\
\hline & Men & $21.7 \pm 3.1$ & $22.6 \pm 2.1$ & $48.0 \pm 5.0$ & $50.7 \pm 5.4$ & $68.8 \pm 5.4$ & $87.0 \pm 5.4$ & $85.0 \pm 4.9$ & $3.32 \pm 0.41$ & $27.4 \pm 3.0$ & $27.5 \pm 2.4$ \\
\hline & $\mathrm{P}^{*}$ & 0.083 & $0.041^{*}$ & $0.002^{*}$ & $0.001^{*}$ & $<0.001^{*}$ & $<0.001^{*}$ & $<0.001^{*}$ & 0.367 & $<0.001^{*}$ & $<0.001^{*}$ \\
\hline \multirow{3}{*}{$\begin{array}{l}\text { Group } 3 \\
n=60\end{array}$} & Women & $21.1 \pm 2.0$ & $22.0 \pm 2.3$ & $44.5 \pm 4.7$ & $47.4 \pm 5.0$ & $60.5 \pm 3.9$ & $76.1 \pm 4.8$ & $74.0 \pm 4.3$ & $3.64 \pm 0.57$ & $23.7 \pm 2.3$ & $23.8 \pm 2.0$ \\
\hline & Men & $21.2 \pm 2.8$ & $22.4 \pm 2.3$ & $46.2 \pm 6.9$ & $47.8 \pm 6.8$ & $67.5 \pm 3.7$ & $86.1 \pm 4.1$ & $83.5 \pm 4.1$ & $3.26 \pm 0.53$ & $26.0 \pm 1.7$ & $26.4 \pm 1.9$ \\
\hline & $\mathrm{P}^{*}$ & 0.912 & 0.547 & 0.248 & 0.803 & $<0.001^{*}$ & $<0.001^{*}$ & $<0.001^{*}$ & $0.011^{*}$ & $<0.001^{*}$ & $<0.001^{*}$ \\
\hline
\end{tabular}

${ }^{*} P<0.05$, Statistically significant.

Table 3: Morphometric measurements of all individuals according to sex (independent of age).

\begin{tabular}{|c|c|c|c|c|c|c|c|c|c|c|c|}
\hline & Gender & NW & $\mathrm{NL}$ & $\mathrm{MCH}$ & $\mathrm{LCH}$ & PCL & aTEA & sTEA & EPAA & ALCSD & AMCSD \\
\hline \multirow{2}{*}{$\begin{array}{l}\bar{\pi} \\
\stackrel{\pi}{0} \\
\end{array}$} & Women & $21.2 \pm 2.3$ & $22.0 \pm 2.2$ & $44.8 \pm 4.4$ & $47.3 \pm 4.6$ & $60.7 \pm 4.9$ & $76.2 \pm 5.9$ & $74.4 \pm 5.6$ & $3.59 \pm 0.67$ & $24.2 \pm 2.9$ & $24.4 \pm 2.7$ \\
\hline & Men & $22.1 \pm 2.9$ & $22.7 \pm 2.2$ & $48.7 \pm 6.1$ & $50.5 \pm 5.9$ & $67.9 \pm 4.4$ & $86.2 \pm 4.6$ & $83.9 \pm 4.3$ & $3.39 \pm 0.58$ & $27.6 \pm 2.8$ & $27.9 \pm 2.8$ \\
\hline & $\mathrm{P}^{*}$ & $0.019^{*}$ & $0.037^{*}$ & $<0.001^{*}$ & $<0.001^{*}$ & $<0.001^{*}$ & $<0.001^{*}$ & $<0.001^{*}$ & $0.037^{*}$ & $<0.001^{*}$ & $<0.001^{*}$ \\
\hline
\end{tabular}

${ }^{*} P<0.05$, Statistically significant.

AP width was $68.1 \pm 7.75 \mathrm{~mm}$. The knee size of the Arabs was generally smaller than that of the Caucasians and larger than that of the Asians. They reported a significant asymmetry in the femoral condyles and proximal tibia plateau of the Arabian knees; the ML and AP values were smaller in women than in men (13). In a morphological study conducted by Lim et al. using MRI, the mean ML width was measured as 81.5 and $76.7 \mathrm{~mm}$, the medial AP as $62.7 \mathrm{~mm}$ and 56.8 $\mathrm{mm}$, and lateral AP as $59.0 \mathrm{~mm}$ and $58.4 \mathrm{~mm}$ in men and women, respectively (14).

In another morphological study based on MRI, Han et al. reported differences in the morphometric parameters of men and women by age and determined smaller distal femoral parameters in older individuals compared with other age groups. The TEA width was found as $83.2 \mathrm{~mm}$ in men and $73.9 \mathrm{~mm}$ in women. They suggested that the ML width determined soft tissue and bone coverage (10). In the present study, aTEA was measured as $86.2 \mathrm{~mm}$ and $76.2 \mathrm{~mm}$, sTEA as $83.9 \mathrm{~mm}$ and $83.9 \mathrm{~mm}, \mathrm{PCL}$ as 67.9 $\mathrm{mm}$ and $60.7 \mathrm{~mm}$, and NW as $22.1 \mathrm{~mm}$ and 21.2 in men and women, respectively.
The sex-based values obtained from the present study indicated larger knee dimensions in men compared with women, as also reported for both Asian and Arab populations. The values were similar to those reported for the individuals living in the AsiaPacific region but were slightly larger compared with those for the Arab population. In addition, similar to the study by Han et al., the present study found that ALCSD, AMCSD, NW, MCH, and LCH decreased in parallel to the increase in age.

It is very important that orthopedic surgeons choose the most appropriate implant for the patient population (11). Orthopedic surgeons and prosthetic manufacturers need to pay more attention to the reproduction of patients' anatomy and personalization of the implant (15). Overhanging of the femoral component causes soft tissue irritation, inadequate ligament balance, stress changes in the patellofemoral joint, and restriction of movement due to pain. However, undersizing of the ML width relative to the femoral condyle can lead to postoperative complications, such as massive hemorrhage, cancellous bone damage, and osteolysis due to submicron particles 
from polyethylene wear $(10,16)$. Such postoperative complications can be prevented by sex-specific prosthesis designs.

Rotational incompatibility in TKA is still responsible for an unacceptably large number of failures. $P C L$ length in the axial plane is in a mean internal rotation of $3^{\circ}$ to STEA and $5^{\circ}$ to aTEA (17). Amaranath et al. suggested that the rotational alignment of the femoral component was one of the important factors for the long-term success of TKA. PCL was in a mean internal rotation of $2.3 \pm 1.8^{\circ}$ to TEA, which was higher in women. Also, the femoral component was traditionally parallel to TEA and in external rotation of $3^{\circ}$ to PCL (18). Further, the angle between aTEA and STEA was examined, which was not considered in previous studies. EPAA was measured as $2.76^{\circ}$ in men and $2.42^{\circ}$ in women, indicating that the STEA length is important for the application of the prosthesis in the correct rotation.

One of the most important limitations of this study is the absence of a morphological evaluation of patients with advanced-stage osteoarthritis. Another limitation was that this was a single-center study and could not generalize the entire population. Furthermore, whether women in the elderly group were in menopause was not known.

In conclusion, the sex-related data in this study were similar to those in Asia-Pacific but slightly larger than those reported for the Arab population. It is believed that the normal reference values of the distal femur may serve as a guide for knee surgery and the design of new knee replacement implant systems.

\section{Compliance with ethical standards}

Conflicts of interest: The authors declare no conflicts of interest and financial support.

Ethical approval: Ethics committee approval was obtained [approval \#2018/2-1].

\section{REFERENCES}

1. Hinman RS, Crossley KM. Patellofemoral joint osteoarthritis: an important subgroup of knee osteoarthritis. Rheumatology 2007;46(7):1057-1062.

2. Loeser RF. Aging and osteoarthritis. Curr Opin Rheumatol. 2011;23(5):492-496.

3. Hunter DJ, SpectorTD. The role of bone metabolism in osteoarthritis. Curr Rheumatol Rep 2003;5(1):15-9.

4. Insall JN, Dorr LD, Scott RD, Scott WN. Rationale of the Knee Society clinical rating system. Clin Orthop Relat Res 1989;(248):13-14.
5. Cheng CK, Lung CY, Lee YM, Huang $\mathrm{CH}$. A new approach of designing the tibial baseplate of total knee prostheses. Clin Biomech 1999;(14):112-117.

6. Yue B, Varadarajan KM, Ai S, Tang T, Rubash HE, Li G. Gender differences in the knees of Chinese population. Knee Surg Sports Traumatol Arthrosc 2011;19(1):80-88.

7. Merchant AC, Arendt EA, Dye SF, Fredericson M, Grelsamer RP, Leadbetter WB, et al. The female knee: anatomic variations and the female-specific total knee design. Clin Orthop Relat Res 2008;(466):3059-3065.

8. Cheng FB, Ji XF, Lai Y, Feng JC, Zheng WX, Sun YF, et al. Three dimensional morphometry of the knee to design the total knee arthroplasty for Chinese population. Knee 2009;16(5):341-347.

9. Bellemans J, Carpentier K, Vandenneucker H, Vanlauwe JJ. The John Insall Award. Both morphotype and gender influence the shape of the knee in patients undergoing TKA. Clin Orthop Relat Res 2010;468(1):29-36.

10. Han H, Oh S, Chang CB, Kang SB. Anthropometric difference of the knee on MRI according to gender and age groups. Surg Radiol Anat 2016;38(2):203-211.

11. Guy SP, Farndon MA, Sidhom S, Al-Lami M, Bennett C, London NJ. Gender differences in distal femoral morphology and the role of gender specific implants in total knee replacement: a prospective clinical study. Knee 2012;19(1):28-31.

12. Yan M, Wang J, Wang Y, Zhang J, Yue B, Zeng Y. Gender-based differences in the dimensions of the femoral trochlea and condyles in the Chinese population: correlation to the risk of femoral component overhang. Knee 2014;21(1):252-256.

13. Hafez MA, Sheikhedrees SM, Saweeres ES. Anthropometry of Arabian Arthritic Knees: Comparison to Other Ethnic Groups and Implant Dimensions. J Arthroplasty 2016;31(5):1109-1116.

14. Lim HC1, Bae JH, Yoon JY, Kim SJ, Kim JG, Lee JM. Gender differences of the morphology of the distal femur and proximal tibia in a Korean population. Knee 2013;20(1):26-30.

15. Morris WZ, Gebhart JJ, Goldberg VM, Wera GD. Implant Size Availability Affects Reproduction of Distal Femoral Anatomy. J Knee Surg 2016;29(5):409-413.

16. Hitt K, Shurman II JR, Greene K, McCarthy J, Moskal J, Hoeman $\mathrm{T}$ et al. Anthropometric measurements of the human knee: correlation to the sizing of current knee arthroplasty systems. J Bone Joint Surg Am 2003;85(4):115-122.

17. Victor J. Rotational alignment of the distal femur: a literature review. Orthop Traumatol Surg Res 2009;95(5):365-372.

18. Amaranath JE, Moopanar TR, Sorial RM. Defining distal femoral anatomy for rotational alignment in total knee arthroplasty: a magnetic resonance imaging-based study. ANZ J Surg 2014;84(11):852-855.

\section{STATEMENT OF AUTHOR'S CONTRIBUTION:}

Each individual author contributed individually and significantly to this study.

BAH (0000-0002-9281-652X) was responsible for the data collection, management, manuscript editing, and figure illustration.

TBH (0000-0001-6500-7260) analyzed and interpreted the data.

KAS (0000-0002-8469-4518) drafted and reviewed the manuscript and contributed to the intellectual concept of the study.

AR (0000-0001-8955-6950) collected the data.

ii (0000-0003-2136-3604) drafted and reviewed the manuscript and contributed to the intellectual concept of the study.

SS (0000-0001-6920-4317) collected the data. 